\title{
Macroinvertebrados bentónicos como bioindicadores de calidad de agua del trópico húmedo en las microcuencas de los alrededores de Bluefields, RAAS
}

\author{
Néstor González A., Sonia Sánchez Mateo \& Álvaro Mairena Valdivia \\ Bluefields Indian \& Caribbean University (BICU)
}

La ciudad de Bluefields está ubicada en la parte oeste de la bahía que lleva su nombre, la cual pertenece al Sistema de Humedales de la Bahía de Bluefields. Este sistema a su vez está conformado por diversos ecosistemas, desde salados a dulceacuícolas, distribuidos en el entorno de la laguna costera. La cuenca de la cual es receptor el sistema es la del río Escondido, con un área total de $12.700 \mathrm{~km}^{2}$. En los alrededores de Bluefields se localizan numerosos nacimientos de agua que recorren la ciudad y que posteriormente desembocan en la Bahía. En esta región, la reducción de cobertura vegetal original se ha acelerado por el crecimiento de la población, lo cual puede afectar en alto grado los cuerpos de agua que nacen allí, perturbando a las comunidades de organismos que en ellos se desarrollan.

Los organismos que habitan en los ríos pueden ser utilizados como indicadores de la calidad del agua, debido a que presentan diferentes rangos de tolerancia a la contaminación de su hábitat (EARTH, 2007). El grupo más utilizado es el de los macroinvertebrados acuáticos (EARTH, 2007), el cual incluye moluscos (caracoles y almejas), crustáceos (camarones y cangrejos), algunas especies de gusanos (nematodos, planarias, anélidos como lombrices), ácaros y un gran número de especies de insectos (larvas y adultos).

La ventaja de utilizar a los macroinvertebrados es que, en principio, no es necesario realizar identificaciones, a nivel de especie, para aplicar los índices, sino que basta con realizar el reconocimiento de grupos taxonómicos más sencillos. Normalmente se identifican a nivel de familias, para lo cual solo se necesitan, como mucho, una lupa manual de campo de diez aumentos, y una guía de macroinvertebrados. Con estas dos herramientas, cualquier persona con los conocimientos necesarios puede ser capaz de realizar una identificación precisa y poder aplicar los índices biológicos (Puig, 1999).

La mayor parte de los investigadores señalan que dentro de los grupos faunísticos que son considerados como bioindicadores de la calidad ambiental, los macroinvertebrados acuáticos son los mejores bioindicadores de la calidad del agua (Arenas, 1993; Barbour et al., 1995; Figueroa, 1999; Alonso et al., 2002; Fenoglio et al., 2002; Hynea \& Maher, 2003; Cain et al., 2004; Leiva, 2004; Alonso \& Camargo, 2005). Los macroinvertebrados bentónicos se encuentran en todo tipo de ambiente acuático de agua dulce, como ríos o lagunas, donde son importantes para el monitoreo de ese ecosistema acuático en particular (Cummnig \& Klug, 1979).

Pero a pesar de su importancia, la información disponible de las comunidades de macroinvertebrados acuáticos en el país (Ecuador) es aún insuficiente (Roldan, 2001), siendo aún más escasa la información publicada respecto a su dinámica en cabeceras y nacimientos de ríos. El mismo fenómeno se presenta en Nicaragua.

\section{METODOLOGIA}

\section{Área de estudio}

El estudio es de tipo descriptivo, con un enfoque cualitativo y cuantitativo. Se llevó a cabo en los alrededores de la ciudad de Bluefields, en cuatro de las 
principales microcuencas, dos de ellas atraviesan por completo la ciudad de Bluefields, Walpatara y el Pool. Los sitios más distantes corresponden a Esconfran (Santa Matilde) en la parte norte y Lunku creek en la parte sur. Se geo referenciaron cada uno de los puntos, y con la ayuda del programa ARC GIS se elaboró la cartografía (Fig. No 1).

\section{Medición de la Temperatura y el pH}

Para evaluar los parámetros fisicoquímicos se registró la temperatura del agua con un termómetro de mercurio con precisión \pm de $0.1{ }^{\circ} \mathrm{C}$. En el caso del $\mathrm{pH}$ se realizó con un equipo portátil de electrodo para muestra de agua.

\section{Parámetros biológicos}

Para establecer la estructura de la comunidad se llevó a cabo la recolección de macroinvertebrados bénticos en diferentes sitios de muestreo. Para la recolección de las muestras se siguió la metodología empleada por Escobar, 1989 y utilizada por Roldán 1999 y Zúñiga et al 1993. En el laboratorio CIAB (Centro de Investigaciones Acuáticas-BICU), se procedió a la identificación de las muestras, con ayuda del estereoscopio hasta el taxón de Orden y Familia con base al soporte bibliográfico (Roldán, 1988; McCafferty, 1983). Se calculó el índice de calidad del agua BMWP'CR, modificado por Costa Rica, y se utilizarán los criterios de la ecología sapróbita (Zúñiga de Cardozo, 2000). (Tabla No 1).

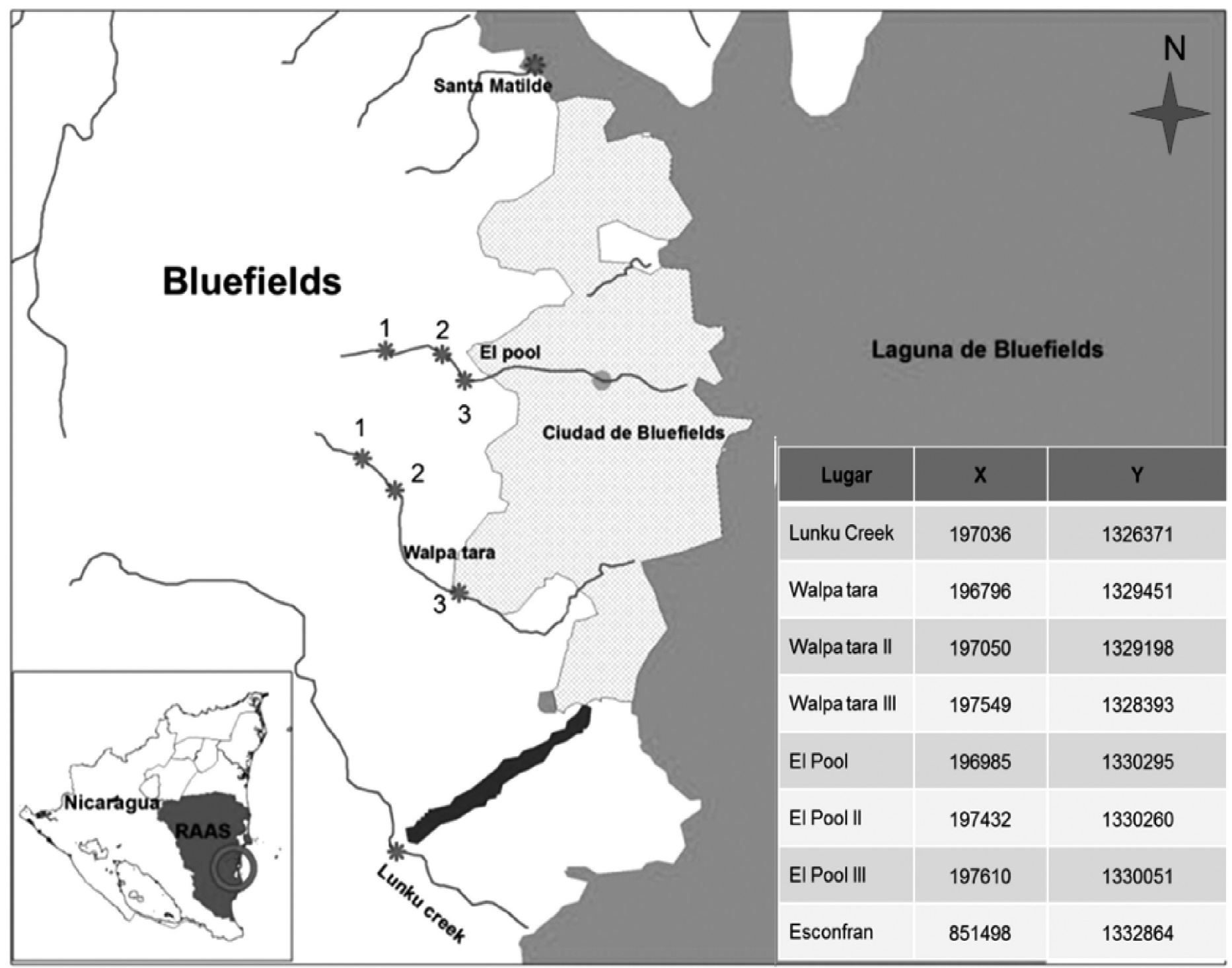

Figura 1. Localización del área de estudio, escala 1: 28,000 (Coordenadas Geográficas en UTM / WGS 84). 
Tabla $N^{0}$ 1. Puntaje y niveles de calidad de agua según $B M W P^{\prime} C R$ "Biological Monitoring Working Party” adaptado para Costa Rica (Springer, M. 2007).

\begin{tabular}{|c|l|c|l|c|}
\hline Clase & \multicolumn{1}{|c|}{ Calidad } & BMWP'CR/Col & \multicolumn{1}{c|}{ Significado } & Color \\
\hline I & Excelente & $>150$ & Aguas de Calidad Excelente & Azul \\
\hline II & Buena & $101-120$ & Aguas muy limpias o limpias & Celeste \\
\hline III & Aceptable & $61-100$ & Aguas ligeramente contaminadas & Verde \\
\hline IV & Dudosa & $36-60$ & Aguas moderadamente contaminadas & Amarillo \\
\hline V & Critica & $16-35$ & Aguas muy contaminadas & Naranja \\
\hline VI & Muy Critica & $<15$ & Aguas fuertemente contaminadas & Rojo \\
\hline
\end{tabular}

\section{Análisis estadísticos}

Para el estudio de las correlaciones se utilizó el método no paramétrico de Spearman y el método paramétrico de Pearson, con la ayuda del programa estadístico SPSS.

El cálculo de los índices de Shannon y Weaver se realizó por familia de macroinvertebrados, con la ayuda de un software el cual computa todos los datos de diversidad que frecuentemente aparecen como indicadores del buen funcionamiento de los ecosistemas. Haciendo uso de la riqueza de familias, es decir, del número de familias y la uniformidad, averiguamos en qué medidas las familias son abundantes.

\section{RESULTADOS Y DISCUSION}

\section{Parámetros físico-químicos}

La temperatura del agua osciló entre los 26 y $30^{\circ} \mathrm{C}$ siendo la temperatura promedio de $28^{\circ} \mathrm{C}$. En general, la temperatura del el agua en los trópicos depende de la altura sobre el nivel del mar (Machado y Roldán, 1981). El promedio del $\mathrm{pH}$ fue de 6.6, nunca superó los 7.3 y tampoco fue inferior a 5.6. En general, el grado de acidez tiene efectos nocivos para los organismos acuáticos e indica una muy posible contaminación del cuerpo de agua (Escobar, 1989). En este caso, el valor de acidez no representa peligro para la biota de la zona. La temperatura $28^{\circ} \mathrm{C}$ y el $\mathrm{pH} 6.6$ permanecieron constantes, con mínimas variaciones entre ligeramente ácidas (5.6) y ligeramente alcalinas (7.3), por lo tanto, no pudo afectar la composición y estructura de las comunidades de MAIA, como tampoco afectó los valores del Índice BMWP y la calidad de agua. De igual forma, la Temperatura no presentó mucha variación en ambas estaciones, por lo que tampoco existe mucha influencia significativa en cuanto a la calidad de agua y el índice BMWP.

\section{Análisis de macroinvertebrados}

En la estación seca se capturó un total de 2,013 individuos entre los cuales fueron reportadas 71 familias y 21 órdenes, sobresaliendo las familias Leptophlebiidae, Culicidae y Thiaridae, con 284, 103 y 92 individuos respectivamente. Los órdenes más abundantes son Ephemeroptera, Trichoptera y Odonata, con 385, 246 y 235 respectivamente. En comparación con la estación lluviosa donde fueron capturados un total de 1,670 individuos perteneciente a 45 familias y 17 órdenes, sobresalieron las familias Baetidae, Hydropsychidae y Leptophlebiidae, con 223, 202 y 137 individuos. Los órdenes más abundantes, al igual que en la estación seca, corresponden a Ephemeroptera, Trichoptera y Odonata, con 367,310 y 242 individuos según el orden con que fueron presentados.

El número de individuos y especies es significativamente más alto en la estación seca que en la estación lluviosa (Jacobsen \& Encalada 1998), similares resultados se obtuvo durante el presente estudio, por lo que es conveniente que los muestreos se realicen en épocas de poca precipitación, y no durante lluvias fuertes ya que estas desfavorecen el registro de dichas especies, porque el hábitat se vuelve muy inestable.

\section{Análisis de calidad biológica por grupos}

Pertenecientes al grupo I "Indicadores de aguas limpias" se encontró un total de 1,270 individuos, que representan un $34.5 \%$ del total de individuos capturados durante los muestreos, y los cuales pertenecen a 17 familias y 10 órdenes. Las familias con mayor número de individuos 
para este grupo son Leptophlebiidae con 421, Baetidae con 299, Helicopsychidae con 155 y Simuliidae con 142 individuos. El orden con mayor número de individuos capturados corresponde a Ephemeroptera con 730 individuos.

Baetidae, Simuliidae y Perlidae son indicadores de aguas limpias y bien oxigenadas (Guerrero-Bolaños, 2003). La mayoría de estos individuos fueron capturados principalmente en las partes altas o cabeceras de Walpatara y Lunku Creek. (Tabla $\mathrm{N}^{\mathrm{o}} 2$ )

Del grupo II "Indicadores de aguas de mediana contaminación” se capturó un total de 1,623 individuos que representan un $44.1 \%$ del total capturados, con 29 familias y 14 órdenes. Las familias con mayor número de individuos son Hydropsychidae con 282, Poecilidae con 174, Hirudinea con 154 y Thiaridae con 143. Aquí los órdenes de Trichoptera y Odonata fueron los más abundantes con 383 y 342 respectivamente.

La familias Poecilidae con 174 individuos y Cichlidae con 69 individuos es tomada en cuenta, ya que según Huidobro (2000), en Vásquez et al, los peces son considerados buenos indicadores de la calidad del medio, por lo que son de gran diversidad y abundancia e indican que es un ambiente sano para todas las demás formas de vida. (Tabla $\mathrm{N}^{\mathrm{o}} 3$ ).

Tabla $\mathbf{N}^{\circ} 2$ Macroinvertebrados indicadores de aguas limpias

\begin{tabular}{|c|c|c|c|c|}
\hline \multicolumn{5}{|c|}{ Índice BMWP (Modificado de Roldán 1999, Zúñiga et al 1993.) } \\
\hline Familia & Orden & Valor & Frecuencia & Grupo \\
\hline Helicopsychidae & Trichoptera & 10 & 155 & \multirow{18}{*}{$\begin{array}{c}\text { Grupo I Indicador de } \\
\text { Aguas Limpias }\end{array}$} \\
\hline Perlidae & Plecóptera & 10 & 15 & \\
\hline Perilestidae & Odonata & 10 & 7 & \\
\hline Psephenidae & Coleóptera & 10 & 47 & \\
\hline Heptageniidae & Ephemeroptera & 10 & 10 & \\
\hline Odontoceridae & Trichoptera & 10 & 1 & \\
\hline Veliidae & Hemíptera & 8 & 94 & \\
\hline Leptophlebiidae & Ephemeroptera & 8 & 421 & \\
\hline Baetidae & Ephemeroptera & 8 & 299 & \\
\hline Simuliidae & Díptera & 8 & 142 & \\
\hline Gyrinidae & Coleóptera & 8 & 1 & \\
\hline Leptoceridae & Trichoptera & 8 & 6 & \\
\hline Corydalidae & Megaloptera & 8 & 29 & \\
\hline Pseudothelpusidae & Crustáceo & 8 & 24 & \\
\hline Glossosomatidae & Trichoptera & 8 & 4 & \\
\hline Calamoceratidae & Trichoptera & 8 & 7 & \\
\hline Blaberidae & Blattodea & 8 & 8 & \\
\hline Total & & & 1270 & \\
\hline
\end{tabular}


Tabla $\mathbf{N}^{\circ} \mathbf{3}$ Macroinvertebrados indicadores de aguas de mediana contaminación

\begin{tabular}{|c|c|c|c|c|}
\hline \multicolumn{5}{|c|}{ Índice BMWP (Modificado de Roldán 1999, Zúñiga et al 1993.) } \\
\hline Familia & Orden & Valor & Frecuencia & Grupo \\
\hline Gomphidae & Odonata & 7 & 16 & \multirow{30}{*}{$\begin{array}{l}\text { Grupo II Indicadores } \\
\text { de aguas de mediana } \\
\text { contaminación }\end{array}$} \\
\hline Platysticitidae & Odonata & 7 & 77 & \\
\hline Lutrochidae & Coleóptera & 7 & 11 & \\
\hline Megapodagrionidae & Odonata & 7 & 130 & \\
\hline Zygoptera & Odonata & 7 & 3 & \\
\hline Himenóptera & Himenóptera & 7 & 12 & \\
\hline Philopotamidae & Trichoptera & 7 & 12 & \\
\hline Libellulidae & Odonata & 6 & 116 & \\
\hline Polycentropodidae & Trichoptera & 6 & 8 & \\
\hline Xiphocentronidae & Trichoptera & 6 & 2 & \\
\hline Elmidae & Coleóptera & 6 & 71 & \\
\hline Tiphocentronidae & Trichoptera & 6 & 3 & \\
\hline Ancylidae & Molusco & 6 & 56 & \\
\hline Pyralidae & Lepidóptera & 5 & 30 & \\
\hline Poecilidae & Piscis & 5 & 174 & \\
\hline Leptohyphidae & Ephemeroptera & 5 & 22 & \\
\hline Hydrobiosidae & Trichoptera & 5 & 62 & \\
\hline Cichlidae & Piscis & 5 & 69 & \\
\hline Hirudinea & Sanguijelas & 5 & 154 & \\
\hline Hydropsychidae & Trichoptera & 5 & 282 & \\
\hline Thiaridae & Molusco & 5 & 143 & \\
\hline Nepidae & Hemíptera & 5 & 3 & \\
\hline Helipsychidae & Trichoptera & 5 & 14 & \\
\hline Dendrobatidae & Anfibio & 5 & 2 & \\
\hline Palaemonidae & Decápoda & 5 & 132 & \\
\hline Dryophidae & Coleóptera & 5 & 6 & \\
\hline Anguilidae & Piscis & 5 & 1 & \\
\hline Aracnidae & Arácnido & 5 & 2 & \\
\hline Aerididae & Ortóptera & 5 & 10 & \\
\hline Total & & & 1623 & \\
\hline
\end{tabular}

Del grupo III "Indicadores de aguas contaminadas" se capturó un total de 793 individuos que equivalen a un $21.4 \%$ del total capturado, con 27 familias y nueve órdenes. Las familias con mayor número de individuos son Culicidae con 169 individuos y Coenagrionidae con 118. Los órdenes con mayor número de individuos son Díptera y Odonata con 217 y 128 respectivamente.

Chironomidae, Culicidae, Oligochaeta y Polichaeta son indicadores de condiciones anaeróbicas y/o de alta contaminación (Guerrero-Bolaños, 2003). Estas familias 
se reportaron en las partes bajas (Walpatara, El Pool y Esconfran). En el caso de Esconfran, durante la estación seca puede reportar salinidad de hasta 15\% (Brenes y Castillo, DIPAL, 1999). (Tabla No 4).

\section{Índice de biodiversidad}

El índice de biodiversidad biológica de Shannon y Weaver, durante todo el estudio presentó un índice de $3.46\left(\mathrm{H}^{\prime}\right)$, con 3,686 individuos capturados $(\mathrm{N})$, con 73 familias capturadas (F) y una uniformidad de $0.8(\mathrm{E})$. Según González, V.(2012) sepuede haceruna aproximación del estado de las aguas con el valor del índice: valores superiores a tres implicarían aguas limpias; valores entre uno y tres, aguas más o menos contaminadas, y valores inferiores a uno, aguas muy contaminadas. Dado lo antes expresado por González V. (2012) tenemos que para la mayoría de los sitios muestreados, en ambas estaciones, los valores se encuentran entre uno y tres, lo que indica aguas más o menos contaminadas, a excepción de la zona

Tabla $\mathbf{N}^{\circ} 4$ Macroinvertebrados indicadores de aguas contaminadas

\begin{tabular}{|c|c|c|c|c|}
\hline \multicolumn{5}{|c|}{ Índice BMWP (Modificado de Roldán 1999, Zúñiga et al 1993.) } \\
\hline Familia & Orden & Valor & Frecuencia & Grupo \\
\hline Anfibios & Larvas de guiri sapos & 4 & 77 & \multirow{28}{*}{$\begin{array}{l}\text { Grupo III Indicador de } \\
\text { aguas contaminadas }\end{array}$} \\
\hline Gerridae & Hemíptera & 4 & 38 & \\
\hline Tipulidae & Díptera & 4 & 6 & \\
\hline Calopterygidae & Odonata & 4 & 10 & \\
\hline Dytiscidae & Coleóptera & 4 & 2 & \\
\hline Naucoridae & Hemíptera & 4 & 14 & \\
\hline Belostomatidae & Hemíptera & 4 & 1 & \\
\hline Noturidae & Coleóptera & 4 & 1 & \\
\hline Hydrometidae & Hemíptera & 4 & 1 & \\
\hline Crasostrea & Bivalvo & 4 & 80 & \\
\hline Coenagrionidae & Odonata & 4 & 118 & \\
\hline Notonectidae & Hemíptera & 4 & 11 & \\
\hline Ceratopogonidae & Anélido & 4 & 3 & \\
\hline Staphylinidae & Coleóptera & 4 & 2 & \\
\hline Gonolidae & Díptera & 4 & 2 & \\
\hline Hydrobiidae & Molusco & 3 & 28 & \\
\hline Physidae & Molusco & 3 & 79 & \\
\hline Glossiphoniidae & Anélido & 3 & 1 & \\
\hline Psychodidae & Díptera & 3 & 1 & \\
\hline Syrphydae & Díptera & 2 & 14 & \\
\hline Chironomidae & Díptera & 2 & 24 & \\
\hline Hyalellidae & Crustáceo & 2 & 2 & \\
\hline Culicidae & Díptera & 2 & 169 & \\
\hline Tubificidae & Anélido & 1 & 13 & \\
\hline Oligochaeta & Anélido & 1 & 34 & \\
\hline Polichaeta & Anélido & 1 & 42 & \\
\hline Tubifex & Anélido & 1 & 20 & \\
\hline Total & & & 793 & \\
\hline
\end{tabular}


del Pool I que durante la estación seca presenta valores superiores a tres, lo cual denota aguas limpias.

En estos casos, la temperatura no es un factor determinante ya que para ambas estaciones la temperatura promedio se mantiene en $28^{\circ} \mathrm{C}$. Jacobsen et al. (1997) sugieren que la temperatura es el principal factor para la riqueza y composición de especies de invertebrados acuáticos. La temperatura en condiciones normales varía la estructura de la comunidad de macroinvertebrados acuáticos, de tal manera que un río con adecuada auto-depuración puede registrar mejoras de calidad en zonas más bajas, pese a la influencia de una contaminación rutinaria.

\section{Niveles de Calidad de Agua}

En comparación con la estación seca es evidente que existe un incremento significativo del valor del Índice BMWP, aunque esto no signifique que la calidad del agua sea mejor. En el caso de Esconfran existe un aumento del valor del Índice en la estación lluviosa, de 36.4 a 52, en comparación con la estación, pero la calidad del agua sigue siendo dudosa (aguas moderadamente contaminadas). Esto se debe a que en esta estación disminuye la salinidad, modificando el hábitat y permitiendo el desarrollo larval de ciertos macroinvertebrados, lo cual se debe al incremento de la precipitación y del volumen de agua que descargan las principales cuencas hidrográficas.

En el caso de Walpatara zona I y II existe un aumento significativo en comparación con la estación seca, no solo incrementando el valor del Índice, sino también la calidad de agua. En la Zona II de Walpatara, el agua pasa de calidad dudosa a calidad aceptable con promedio de 55.5 a 73.3 (de aguas moderadamente contaminadas a aguas ligeramente contaminadas). En la zona III ocurre lo contrario, es decir, el valor del índice disminuye, haciendo que también la calidad del agua disminuya. En este sentido, el agua pasa de calidad dudosa, con promedio de 38.3 (aguas moderadamente contaminadas), a aguas de calidad crítica con promedio de 30 (aguas muy contaminadas).

Para la zona del Pool la situación cambia, principalmente en las zonas II y III, respecto a la estación. En invierno, la calidad del agua es ligeramente superior a la estación seca, al pasar de calidad dudosa a crítica (de aguas moderadamente contaminadas a aguas muy contaminadas). Manteniendo aguas de calidad aceptable (aguas ligeramente contaminadas) en ambas estaciones.

Todo esto se debe a que los macroinvertebrados responden a los cambios ambientales más rápidamente que los vertebrados utilizados como bioindicadores de calidad de agua los cuales pueden exhibir respuestas evidentes cuando ya es tarde, desde el punto de vista del manejo de la conservación (Zúñiga de Cardozo, 2000).

\section{Correlación Paramétrica de Pearson}

Según esta correlación de Pearson en función a las varianzas entre las variables BMWP y $\mathrm{pH}$, la correlación es moderada, con valor de $50.5 \%$, es decir, una correlación media; entre BMWP y Temperatura, la correlación es inversamente proporcional, con valor de (-61.7\%), es decir, a menor variación de Temperatura mayor Índice BMWP. También existe una correlación inversamente proporcional entre la Temperatura y el $\mathrm{pH}$ de (-29\%), es decir, que a menor variación del $\mathrm{pH}$, mayor Temperatura. En cuanto a la relación del Índice BMWP y la Estación, la correlación es muy débil, con un valor de $15.7 \%$, es decir, que los valores de los Índices de biodiversidad no varían mucho de una estación a otra. (Tabla $\mathrm{N}^{\mathrm{o}}$ 5).

Tabla N 5 Correlación paramétricas de Pearson

\begin{tabular}{|l|l|l|l|l|l|}
\hline \multicolumn{7}{|c|}{ Correlaciones de Pearson } & Temperatura & Estación \\
\hline BMWP & & \multicolumn{1}{|c|}{ BMWP } & pH & .157 \\
\hline $\mathbf{p H}$ & Correlación de Pearson & 1 & $.505^{* *}$ & $-.617^{* *}$ & $.280^{*}$ \\
\hline Temperatura & Correlación de Pearson & $.505^{* *}$ & 1 & $-.290^{*}$ & -.043 \\
\hline Estación & Correlación de Pearson & $-.617^{* *}$ & $-.290^{*}$ & 1 & 1 \\
\hline $\begin{array}{l}* * \text { La correlación es significativa al nivel 0,01 (bilateral). } \\
* . \text { La correlación es significante al nivel 0,05 (bilateral). }\end{array}$ & $.280^{*}$ & -.043 & \\
\hline
\end{tabular}


En este sentido, las características físico-químicas, como $\mathrm{pH}$ y Temperatura, son las que suelen ejercer una importante influencia sobre la distribución de los macroinvertebrados fluviales (Vivas et al, 2002) y son a menudo los parámetros a los cuales los organismos son más sensibles (Roldan, 1992). De esta forma, en los tramos donde se detectan problemas pueden establecerse medidas para detectar las posibles fuentes de dichas alteraciones, y por ello, el uso de indicadores biológicos representa una herramienta útil y rápida para la evaluación de la calidad de las aguas (Ozcos et al., 2006).

\section{Correlación No Paramétrica de Spearman}

Según Spearman, la correlación entre BMWP y pH es media (57.2\%). Es decir, que aquí el Índice BMWP no aumenta ni disminuye; es inversamente proporcional entre el Índice BMWP y Temperatura, con $-64.7 \%$; entre $\mathrm{pH}$ y Temperatura, con $-37.5 \%$, y entre la Temperatura y la Estación, con $-47 \%$. Con lo cual se deduce que a menor variación de Temperatura es mayor el Índice de BMWP, y que, a menor variación de la Temperatura, el pH es más estable, es decir, tanto la Temperatura puede inferir sobre el $\mathrm{pH}$, si ésta se combina con otros factores externos, y que la correlación es muy débil en cuanto a la Estación y la Temperatura, porque no existe mucha variación de Temperatura de acuerdo a la Estación. La correlación es débil entre la Estación y el Índice BMWP, con $16.6 \%$, por tanto, muy poco influye la Estación seca o lluviosa con el aumento o disminución del Índice BMWP. (Tabla Nº 6).

La composición y diversidad de la fauna de los macroinvertebrados acuáticos puede mostrar una gran variabilidad en condiciones naturales $y$ esto dependerá de las características físicas, climáticas y geomorfológicas en las cuales reside (Barbour et al., 1999; Green \& Swietlik, 2000). En este estudio fueron observadas diferencias evidentes en la composición de las comunidades de macroinvertebrados, provocadas más por el impacto de las actividades humanas desarrolladas en estas microcuencas, que por las condiciones naturales, principalmente en las zonas medias y bajas.

Jacobsen et al. (1997) sugieren que la temperatura es el factor mayor para la riqueza y composición de especies de invertebrados acuáticos. La temperatura en condiciones normales varía la estructura de la comunidad de macroinvertebrados acuáticos, donde un río con adecuada auto-depuración puede registrar mejora de calidad en zonas más bajas pese a la influencia de una contaminación rutinaria.

\section{Zonas críticas}

Las zonas más críticas tanto en la estación seca como lluviosa resultan ser las partes media y baja de las microcuencas de Walpatara y el Pool. En la zona II de Walpatara (Posa del Paso) y zona III (Puente San Pedro), los resultados indican aguas de calidad dudosa, es decir, moderadamente contaminadas (Índice BMWP) durante la estación lluviosa. En la zona del Pool, además de los problemas de la erosión y deforestación de los alrededores, se le suman los problemas de las aguas residuales de toda la población que se asienta a escasos metros de este cauce natural, y que vierten directamente los desechos sólidos y orgánicos sin tratamiento.

Tabla $\mathbf{N}^{\circ} 6$ Correlación no paramétricas de Spearman

\begin{tabular}{|l|l|l|l|l|l|}
\hline \multicolumn{5}{|c|}{ Correlaciones de Spearman } \\
\hline \multicolumn{1}{|c|}{ Rho de Spearman } & & \multicolumn{1}{|c|}{ BMWP } & pH & Temperatura & Estación \\
\hline BMWP & Coeficiente de correlación & 1.000 & $.572^{* *}$ & $-.647^{* *}$ & .166 \\
\hline $\mathrm{pH}$ & Coeficiente de correlación & $.572^{* *}$ & 1.000 & $-.375^{* *}$ & $.249^{*}$ \\
\hline Temperatura & Coeficiente de correlación & $-.647^{* *}$ & $-.375^{* *}$ & 1.000 & -.047 \\
\hline Estación & Coeficiente de correlación & .166 & $.249^{*}$ & -.047 & 1.000 \\
\hline $\begin{array}{l}* * \text { La correlación es significativa al nivel 0,01 (bilateral). } \\
* . \text { La correlación es significativa al nivel 0,05 (bilateral). }\end{array}$ & & \\
\hline
\end{tabular}




\section{Posibles Soluciones}

$\checkmark$ Reforestación de los alrededores de estas microcuencas y de su cabecera.

$\checkmark$ Ejecución de un proyecto de reciclaje de desechos sólidos y orgánicos, además de la construcción de un sistema para el manejo de aguas negras.

$\checkmark \quad$ Implementar un plan de ordenamiento urbanístico y rural.

$\checkmark$ Eliminar los basureros clandestinos en los alrededores de las microcuencas.

$\checkmark$ Establecer mecanismos que regulen la construcción de las pilas sépticas y servicio higiénicos sin control.

$\checkmark$ Programas de educación ambiental para la concientización de la población en general.

Fomentar la aplicación del uso de índices biológicos como factor decisivo en la toma de decisiones, y no regirse únicamente por los indicadores físicos-químicos del agua.

\section{Recomendaciones}

Deben realizarse, además de los estudios de las poblaciones bénticas, monitoreo constante de todas las variables físico-químicas de la zona y análisis microbiológicos del agua, para poderlos correlacionar con los resultados obtenidos. Así mismo realizar estudios sobre la relación entre la estructura de las comunidades, las variaciones climáticas que se presentan en la zona y cómo el crecimiento de la población urbana y sus actividades cotidianas afectan el comportamiento de los macroinvertebrados en esa zona.

Fomentar la aplicación del uso de índices biológicos como factor decisivo en la toma de decisiones, y no regirse únicamente por los indicadores físicos-químicos del agua.

\section{AGRADECIMIENTO}

Un especial agradecimiento al Centro de Investigaciones Acuáticas y Biológicas de la BICU, a través del MSc. Billy Ebanks, por su apoyo y disposición. Finalmente, a los fondos de investigación de la BICU por el apoyo brindado para que la investigación pudiera llevarse a cabo. 


\section{BIBLIOGRAFIA}

Alonso A., De la Puente M. \& Camargo J.A. (2002). Valoración de los efectos de la contaminación orgánica sobre la comunidad de macroinvertebrados bentónicos en la cabecera del río Henares (Guadalajara, España). XI Congreso de la Asociación Española de Limnología y III Congreso Ibérico de Limnología. Madrid 17-21 Junio.

Alonso A. \& Camargo J. A. (2005). "Estado actual y perspectivas en el empleo de la comunidad de macroinvertebrados bentónicos como indicadora del estado ecológico de los Ecosistemas fluviales españoles”. Ecosistemas. 3: 1-12.

Arenas J. (1993). Macroinvertebrados bentónicos como bioindicadores de la calidad del agua del río Bío Bío, Chile. Tesis de Doctorado de la Facultad de Ciencias, Universidad de Concepción. 111pp.

Barbour M. T., Gerritsen J., Snyder B. D. \& Stribling J. B. (1995). "Revision to Rapid Bioassessment Protocols for use in stream and rivers: Periphyton, benthic macro invertebrates and fish”. EPA 841_D_97-002.

Barbour, M. T., Gerrisen, B. Snyder \& S. James. (1999). Rapid Bioassessment Protocol For Use in Streams and Wadeable Rivers: Periphyton, Benthic Macroinvertebrates and Fish (Second Ed.). Washington, DC 20460: U.S. Environmental Proteccion Agency; Office of Water.

Boada, M., Mayo, S. i Maneja, R. (coords.): "Els sistemes socioecològics de la conca de la Tordera”. Barcelona: Institució Catalana d'Història Natural. 345-374. ISBN: 978-84-7283-983-0.

Brenes, C. \& Castillo, E. (1999). Hidrografia de la Bahía de Bluefields. Proyecto DIPAL. Bluefields/Haulover, RAAS, Nicaragua.

Cain D., Luoma S. \& Wallace W. (2004). "Linking metal bioaccumulation of aquatic insect to their distribution patterns in mining-impacted river”. Environ. Toxicol. and Chem. 23:1463-1473.

Cummnig K. \& Klug J. (1979). "Feeding ecology of stream invertebrates". Annual Review Ecology and Systemic. 10: 147-172.

Escobar, A.1989. "Estudio de las comunidades macro bentónicas en el río Manzanares y sus principales afluentes y su relación con la calidad de agua”. Actualidades Biológicas, 18(65) 45 - 60.

Fenoglio S., Badino G. \& Bona F. (2002). "Benthic macroinvertebrate communities as indicators of river environment quality: an experience in Nicaragua". Rev. Biol. Trop. 50: 1125-1131.

Figueroa R. (1999). Macroinvertebrados bentónicos como indicadores biológicos de calidad de agua, Río Damas, Osorno, X Región de los Lagos, Chile.Tesis para optar al Magister en Ciencias mención en Zoología. 105 pp.

Gerrero- Bolaños, F. (2003). Los macroinvertebrados bentónicos de pozo azul (cuenca del río gaira, Colombia) y su relación con la calidad del agua. Programa de Biología, Facultad de Ciencias Básicas, Universidad del Magdalena, Santa Marta, Magdalena, Colombia.

González, V (2012). Art. Los macroinvertebrados como bioindicadores. 30 de octubre 2012.

Green, J, \& W. Swietlik. (2000). A Stream Contition Index (SCI) for West Virginia wadeables stream. Tetra Tech: 80 pp.

Hynea R. \& Maher W. (2003). "Invertebrate biomarkers: links to toxicosis that predict population decline". Ecotoxicol. Environ. Safety. 54: 366-374.

Huidobro, C. L. (2000). "Organismos Indicadores de la Calidad del Agua y de la Contaminación (Bioindicadores)”. Peces. p. 195-263. 
Jacobsen, D. Schultz, R. \& Encalada, A. (1997). "Structure and diversity of stream invertebrate communites: the influence of temperature with latitude and altitude". Freshwater Biological Laboratory. University of Copenhagen. Arch. Biol. 38 : 247-262.

Jacobsen, D., A. Encalada. (1998). "The macroinvertebrate fauna of Ecuatorian higlan streams in the wet and dry season". Freshwater Biological Laboratory. University of Copenhagen. Arch. Hydrobiol. 142: 53-70

Leiva J. (2004). Macroinvertebrados bentónicos como bioindicadores de calidad de agua en la Cuenca del estero Peu Peu Comuna de Lautaro IX Región de la Araucania. Tesis presentada a la facultad de Ciencias de la Universidad Católica de Temuco para optar al Grado de Licenciado en Recursos Naturales. Temuco, Chile. 120pp.

MACHADO, T.; ROLDÁN, G. (1981). "Estudio de las características fisicoquímicas y biológicas del río Anorí y sus principales afluentes”. Actualidades Biológicas, 10 (35) 3 - 19.5454 Artículo.

MCCAFFEERTY, P.(1983). Aquatic Entomology. Jones and Bartlett Publishes, Inc.Boston, U.S.A.

Mónica Springer (UCR), Danny Vásquez (UCR), Arturo Castro (UCR), Bert Kohlmann (EARTH). (2007). Bioindicadores de la Calidad de Agua. Derechos reservados Univ. EARTH.

Oscoz, J., F. Campos \& M. C. Escala. (2006). "Variación de las comunidades de Macroinvertebrados bentónicos en relación con la calidad de agua”. Limnetica 25 (3): 683-692.

Puig, M. A., (1999). Los Macroinvertebrados de los Ríos Catalanes. Guía Ilustrada. Primera Edición, 251 pp. Edigraf S.A. España.

Roldan, G.(1988). Guía para el estudio de los macroinvertebrados acuáticos del departamento de Antioquia. FEN Colombia. Colciencias. Universidad de Antioquia.

Roldan, G. (1992). Fundamentos de Limnología Tropical. Colección Ciencia y Tecnología Universitaria de Antioquia. Editorial Universidad de Antioquia. Volumen 1, paginas 529. Medellín, Colombia.

Roldan, G. (1999). "Los macroinvertebrados y su valor como bioindicadores de la calidad del agua. Rev. Acad. Colom. Cienc. 23(88): 375 - 387.

Roldan, G. (2001). Bioindicación de la calidad del agua en Colombia. Propuesta para el uso del método BMWP/Col. Ciencia y Tecnología. Universidad de Antioquia. Medellín. 182 pp.

Roldán G. (2003). Bioindicación de la calidad del agua en Colombia. Editorial Universidad deAntioquia. Colombia. 170pp.

Vivas, S., J. Casas, I. Pardo, S. Robles, N. Bonadas, A. Mellado, N. Prat, J. Alba-Tercedor, M. Álvarez, M. M. Bayo, P. Jaimez-Cuellar, M. L. Suarez, M. Toro, M. R. Vidal-Abarca, C. Zamora-Muñoz \& G. Moya. (2002). "Aproximación multivariante en la exploración de la tolerancia ambiental de las familias de macroinvertebrados de los ríos mediterráneos del proyecto GUADALMED”. Limnetica 21: 149-173.

Zúñiga de Cardoso M., Rojas A. \& Caicedo G. (1993). Indicadores ambientales de calidad de agua en la Cuenca del río Cauca. Asociación de Ingenieros Sanitarios de Antioquia Medellín, Colombia. 2:17-28.

Zúñiga de Cardoso, (2000). Los insectos como bioindicadores de la calidad del agua. Manuscrito. Universidad del Valle. Departamento de Procesos Químicos y Biológicos. Cali, Colombia. 